\title{
Philosophiques
}

\section{Présence de la pensée de Gabriel Marcel au Canada (1940-1978)}

\section{Simonne Plourde}

Volume 6, numéro 1, avril 1979

URI : https://id.erudit.org/iderudit/203112ar

DOI : https://doi.org/10.7202/203112ar

Aller au sommaire du numéro

Éditeur(s)

Société de philosophie du Québec

ISSN

0316-2923 (imprimé)

1492-1391 (numérique)

Découvrir la revue

Citer cet article

Plourde, S. (1979). Présence de la pensée de Gabriel Marcel au Canada (1940-1978). Philosophiques, 6(1), 147-173. https://doi.org/10.7202/203112ar d'utilisation que vous pouvez consulter en ligne.

https://apropos.erudit.org/fr/usagers/politique-dutilisation/ 
BULLETIN

\section{PRÉSENCE DE LA PENSÉE \\ DE GABRIEL MARCEL AU CANADA (1940-1978)}

par Simonne Plourde

Il y a quelque vingt ans, M-M. Davy consacrait à Gabriel Marcel une étude intitulée: Un philosopbe itinérant, Gabriel Marcel $^{1}$. Dans la préface de ce livre, après avoir affirmé que Gabriel Marcel «se présente comme l'un des plus grands penseurs de notre temps ", l'auteur ajoute :

Ses ouvrages et ses pièces de thêâtre sont lus dans toutes les langues. Les thèses et les études sur sa pensée émanent de professeurs et d'étudiants anglais, américains, allemands, italiens, espagnols, japonais ${ }^{2}$

Depuis vingt ans, la liste des thèses et des études consacrées à l'œuvre de Gabriel Marcel s'est de beaucoup accrue. Les bibliographies permettent de jauger l'apport des professeurs et étudiants canadiens, hollandais, hindous, vietnamiens ... ${ }^{3}$

Quel intérêt les Canadiens ont-ils, pour leur part, porté à l'œuvre de Gabriel Marcel et quel accueil ont-ils réservé à sa pensée? Pour répondre à cette question, j’ai voulu dresser l'inventaire le plus exhaustif possible des études canadiennes consacrées à l'œuvre du philosophe français, de 1940 à $1978^{4}$.

1. M.-M. DAvy, Un Pbilosopbe itinérant, Gabriel Marcel, Paris, Flammarion, 1959.

2. Ibid., p. 7 .

3. Cf. Roger Troisfontaines, De l'Existence à l'Être. La Pbilosophie de Gabriel Marcel, Louvain/Paris, Nauwelaearts, deuxième édition, 1968, p. 381-464.

Cf. François et Claire LAPoINTE, Gabriel Marcel and His Critics : An International Bibliography, New York \& London, Garland Publishing, Inc., 1977, 287 p.

4. Cf. en appendice, A) Sources utilisées. 
Il m'a semblé que la somme des études consacrées à la pensée marcellienne me fournirait vraisemblablement le critère d'intérêt et que l'accord exprimé avec sa philosophie, ou tout au moins la valeur reconnue à son auvre, m'indiqueraient l'accueil réservé à Gabriel Marcel au Canada. suivant :

Je présenterai les fruits de ma recherche dans l'ordre

1. Les articles parus dans les revues canadiennes.

2. Les thèses présentées dans les universités canadiennes.

3. Les livres consacrés à l'œuvre de Gabriel Marcel.

4. Les articles de journaux parus au Canada, les émissions radiophoniques, les associations qui font connaître l'œuvre de Gabriel Marcel.

\section{LES REVUES CANADIENNES}

Les articles parus dans les revues canadiennes peuvent être divisés en deux catégories: a) les articles écrits par Gabriel Marcel ; b) les articles qui portent sur Gabriel Marcel et son œuvre.

\section{a) Articles de Gabriel Marcel}

La Nouvelle Releve publiait en 1946 un long article signé par Gabriel Marcel : "Philosophie de l'Épuration, contribution à une théorie de l'hypocrisie dans l'ordre politique ${ }^{5}$. Cette revue était éditée à Montréal et dirigée par MM. Robert Charbonneau et Claude Hurtubise. Une note, à la fin du texte, indique qu'il s'agissait d'un texte inédit, publié avec autorisation verbale donnée par Gabriel Marcel à M. Marcel Raymond.

La Nouvelle Revue Canadienne offrait à ses lecteurs, dans son numéro de septembre-octobre 1951, un article également signé par Gabriel Marcel : "Sartre, Anouilh et le problème de

5. Gabriel MARcel, « Philosophie de l'Epuration, contribution à une théorie de l'hypocrisie dans l'ordre politique ", dans La Nowtelle Releve, vol. IV, $\mathrm{n}^{\circ} 7$ (janvier 1946), p. $559-588 ; n^{\circ} 8$ (février 1946), p. 684-703. 
Dieu $^{6}$ ". C'était une critique théâtrale écrite à l'occasion de la pièce de J.P. Sartre : Le Diable et le Bon Dieu. Rien n'indique l'origine de cet article.

La Revue de l'Université Laval publiait à son tour, en décembre 1963, un article de Gabriel Marcel : «Les méditations d'Amédée Ponceau ${ }^{7} »$. La revue était alors dirigée par $M$. Émile Bégin.

La revue Sciences ecclésiastiques, de Montréal, reproduisait en 1964 un article de Gabriel Marcel paru dans la Revue des Sciences religieuses, de Strasbourg. Le texte porte le titre suivant : « L' 'Initiation philosophique' d'Amédée Ponceau ${ }^{8}$ ».

Il est assez étonnant de trouver ces textes dus à la plume de Gabriel Marcel dans des revues canadiennes. Quelles circonstances ont amené ces publications ? Ont-ils été demandés à l'auteur par des amis? Les indications recueillies sont trop minces pour permettre d'apporter une réponse vraisemblable à ces questions.

\section{b) Articles sur Gabriel Marcel et son œeuvre}

Les revues canadiennes ont, depuis 1940, publié un certain nombre d'études critiques et de comptes rendus ayant pour objet soit l'œuvre de Gabriel Marcel, soit l'existentialisme; dans ce dernier cas, il ne sera fait mention que des textes qui consacrent une partie assez importante à la pensée marcellienne. Ces articles sont présentés ici dans leur ordre chronologique de parution.

En 1941, Joseph-Henri Paul publie dans La Nouvelle Releve une étude intitulée: "La Philosophie de Gabriel Marcel $^{9} »$.

6. Gabriel Marcel, "Sartre, Anouilh et le problème de Dieu ", dans La Nouvelle Revue Canadienne, sept.-oct. 1951 , p. 30-38.

7. Gabriel MARCEL, "Les Méditations d'Amédée Ponceau ", dans Revue de l'Université Laval, vol. XVIII, n 4, (déc. 1963), p. 333-336.

8. Gabriel Marcel, "L' 'Initiation philosophique' d'Amédée, Ponceau ", dans Sciences Ecclésiastiques, vol. XVI, $\mathrm{n}^{\circ} 3$, p. 377-385.

9. J.H. Paul, "La philosophie de Gabriel Marcel ", dans La Nouvelle Releve, $\mathrm{n}^{\circ} 1$ (sept. 1941) p. 19-29. Je regrette de n'avoir pu, jusqu’à ce jour, prendre connaissance de ce texte; je ne puis en présenter ici une analyse. 
En 1949, la Revue de l'Université Laval présente à ses lecteurs un article signé par Arcade $M$. Monette et intitulé «Le Regard en arrière de Gabriel Marcel ${ }^{10}$ ». À l'occasion de la parution du Regard en arrière dans le collectif Existentialisme chrétien, l'auteur passe en revue la pensée marcellienne. Sa présentation est positive. Il indique la direction d'une pensée qui aboutit à une ontologie de l'existence et invite les professeurs à orienter les jeunes élèves en philosophie, non vers « ce qui est vide et séparé », mais vers « ce qui est concret et riche de contenu : l'acte d'exister" (p. 36). "La philosophie, écrit-il, n'est pas seulement une matière à apprendre, c'est bien davantage un univers qui attend ses créateurs /. . / Rassembler dans sa vie intellectuelle essence et existence, c'est recueillir l'être d'abord fragmenté pour remonter en lui et par lui jusqu'au suprême acte d'exister » (p. 36).

Le troisième article recueilli ne porte pas sur Gabriel Marcel, mais il y est question du philosophe français dans une étude qui s'intitule: «Pourquoi l'existentialisme est-il athée $^{11}$ ? ». L'auteur, Benoît Pruche, y situe Gabriel Marcel à l'extrême droite de l'existentialisme et souligne le caractère très chrétien de sa pensée (p. 291).

En 1952, Arcade M. Monette signe un autre article qui, bien qu'il n'analyse pas exclusivement la philosophie de Gabriel Marcel, lui consacre plusieurs pages. Paru dans la Revue de l'Université d'Ottawa, l'article s'intitule: "L'Être, l'exister et Dieu ${ }^{12} "$. L'auteur tente de clarifier les notions d'être et d'existence, utilise une approche de l'être à partir de la phénoménologie marcellienne avant d'aborder une approche proprement spéculative de l'être et de l'exister. Il tire ensuite ses conclusions. À son avis, Gabriel Marcel, en réagissant contre l'idéalisme, a réalisé la loi de l'excès opposé. "L'idéalisme, écrit-il, est un abus intellectuel, mais s'en remettre aux

10. Arcade-M. MONETTE, "Le 'Regard en arrière' de Gabriel Marcel ", dans Revue de l'Université Laval, vol. IV, $\mathrm{n}^{\circ}$ I (septembre 1949), p. 24-37. L'auteur était professeur au Collège dominicain d'Ottawa et chargé de cours à l'Université de Montréal.

11. Benoît Pruche, "Pourquoi l'existentialisme est-il athée ?", dans Revue de l'Université d'Ottawa, vol. XXI, 1951, p. 287-306.

12. Arcade-M. MONETTE, "L'Être, l'exister et Dieu ", dans Revue de l'Université d'Ottawa, vol. XXII, p. 17 1-188. 
puissances de l'amour pour résoudre les problèmes de l'entendement est un contre-excès $»(p .179)$. Il concède quand même que la pensée marcellienne « témoigne d'une intuition de l'être en tant qu'être, bien que le préjugé idéaliste l'empêche d'expliciter conceptuellement sa démarche $\gg$ (p. 182). À propos de la décision marcellienne de fidélité à l'être, Arcade Monette ajoute en note : «Et c'est pourquoi la philosophie de Gabriel Marcel qui trouve là son plus bel aboutissant nous paraît un sommet au milieu des autres philosophies existentielles (p. 187, note 16). La critique de la pensée marcellienne effectuée par l'Auteur trouve appui manifestement sur des catégories thomistes comme en font foi le texte et les références. À travers cette critique, on peut déceler une certaine sympathie pour la pensée du philosophe français.

En avril 1956, Gabriel Marcel venait au Canada. Le 19 avril, l'Université d'Ottawa l'accueillait. La Revue de l'Université d'Ottawa a publié le texte de Jacques Croteau, chargé de présenter au public le philosophe invité. Le texte est intitulé : "Gabriel Marcel, homme de communion ${ }^{13}$ ". Les circonstances invitaient l'Auteur à une esquisse positive de l'œuvre marcellienne; il la réussit fort bien, sans omettre de brèves références à la philosophie thomiste. Il dit ne pas voir d' "opposition fondamentale entre une pensée concrète et un intellectualisme thomiste", mais percevoir "au contraire, deux dimensions complémentaires d'une vision totale de l'être " (p. 496). La pensée marcellienne vient " prolonger des voies déjà ouvertes largement par saint Thomas sur l'existence concrète » (p. 496). "En vertu même de ses aspirations naturelles, la philosophie thomiste ne saurait faire qu'un accueil empressé et sympathique à une pensée chrétienne concrète » (p. 497).

Dans son numéro de juin 1956, la Revue de l'Université Laval publie un commentaire de l'un de ses professeurs de

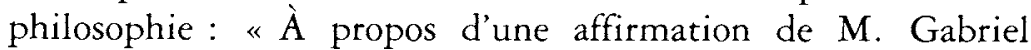
Marcel ${ }^{14}$. Alphonse Saint-Jacques y relève une affirmation

13. Jacques Croteau, o.m.i., "Gabriel Marcel, homme de communion », dans Revue de l'Université d'Ottawa, vol. XXVI (1956), p. 491-497.

14. Alphonse SAINT-JACQues, "À propos d'une affirmation de M. Gabriel Marcel ", dans La Revue de l'Université Laval, vol. X, $\mathrm{n}^{\circ} 10$ (juin 1956), p. 879-887. 
du «réputé philosophe et auteur dramatique français", affirmation entendue à l'émission Conférence de presse de RadioCanada, du 9 avril 1956. Gabriel Marcel avait affirmé, "en réponse à une question d'un de ses interlocuteurs, que l'Église catholique outrepasse ses droits quand elle se mêle de donner aux catholiques des directives concernant la philosophie » (p. 879). À propos de cette affirmation, A. Saint-Jacques pose les questions suivantes : «est-il vrai que l'Église outrepasse ses droits quand elle donne aux catholiques des directives précises en matière de philosophie ? Et dans quelle mesure sommesnous tenus de nous soumettre à ces directives? $\gg(p .880$ ). L'Auteur du commentaire examine le problème dans un texte qu'il appuie principalement sur l'enseignement de saint Augustin, de saint Thomas et des encycliques catholiques. Il conclut que la philosophie ayant des services à rendre à la foi, "l'autorité infaillible du magistère de l'Église ne saurait s'étendre universellement à tout le champ des vérités naturelles, mais seulement à celles qui touchent, de près ou de loin, au domaine des vérités révélées » (p. 883-884). De plus, «c'est à l'Église elle-même qu'il appartient uniquement de juger jusqu'où exactement s'étend la juridiction de son magistère $\gg(p .884)$, et non à chacun de nous de décider de la légitimité de ses enseignements. Il rappelle en terminant que l'Église a réprouvé et proscrit la proposition suivante : "Le dépôt de la foi ne contenant que des vérités révélées, il n'appartient sous aucun rapport à l'Église de porter un jugement sur les assertions des sciences humaines» (p. 884). "Il nous semble, conclut-il, qu'il n'y a entre cette proposition et celle que nous venons d'examiner aucune différence essentielle $\gg($ p. 885).

En 1957, la revue University of Toronto Quarterly publie un article de John Wild intitulé : «Existentialism : a new view of $\operatorname{man}^{15}$ ". Dans ce texte, l'Auteur se réfere assez brièvement à l'existentialisme de Gabriel Marcel qu'il situe parmi les autres philosophies existentialistes. En conclusion, il donne une appréciation globale positive de la philosophie existentielle

15. John WILD, "Existentialism : a new view of man ", dans University of Toronto Quarterly, vol. XXVII, n 1, p. $79-95$ 
dont il reconnaît la valeur et prévoit la place qu'elle occupera dans l'histoire : «... this is a radically new philosophy beyond both idealism and realism. It is a living mode of thought that applies to the problems of living men, and expresses their implicit feeling for life and their place in human history. In its profounder manifestations, as in Kierkegaard and Heidegger, it has achieved insights the significance of which will probably extend far beyond our own time and place in history $\gg$ (p. 95).

En 1959, Jacques Croteau présente aux lecteurs de la Revue de l'Université d'Ottawa une étude sur l'existentialisme. Intitulé «Introduction à l'existentialisme ${ }^{16}$ ", l'article fait le point sur la valeur de cette philosophie nouvelle qui « fait figure plutôt d'un ensemble d'idées, de points de vue, de quelque chose comme un message, un témoignage ou mieux encore une attitude sur l'homme concret en tant qu'engagé dans la réalisation de sa destinée humaine " (p. 90). L'Auteur pose la question : "Sommes-nous justifiés, malgré ses lacunes, de prêter une oreille attentive et bienveillante aux enseignements de l'existentialisme? " (p. 95-96). La réponse est nuancée ; les auteurs existentialistes, écrit-il, "ont le mérite incontesté de rappeler aux chrétiens le sens fondamental du christianisme ; aux métaphysiciens, le souci du concret existant, et cela dans le ton d'une actualité plus éloquente à des oreilles du vingtième siècle. L'encyclique Humani Generis nous conseille une attitude sympathique " (p. 96). En effet, l'encyclique Humani Generis recommandait de chercher l'élément de vérité contenu dans les affirmations fausses elles-mêmes et d'étudier les théories nouvelles, car "les maux ne se soignent bien que s'ils sont préalablement connus" (p. 96). Ces précisions apportées, l'Auteur analyse le problème de l'existentialisme, du point de vue philosophique; il se réfere en cours de route à l'ontologie marcellienne et en exploite la distinction entre "mystère » et "problème " (p. 102-106). Cette distinction lui semble une référence au domaine religieux, «nouvelles catégories bien proches du vocabulaire

16. Jacques CroteaU, o.m.i., "Introduction à l'existentialisme ", dans Revue de l'Université d'Ottawa, vol. XXII, 1959, p. 90-110. 
mystique d'un saint Jean de la Croix . . ${ }^{17} »$. L'existentialisme renvoie au domaine de la subjectivité. Mais «le thomisme n'en veut donc pas à la subjectivité ; il lui assigne de plus sa place dans les divers modes de la connaissance. Mais jamais il n'en fera comme tel un procédé philosophique, puisque, qu'on le veuille ou non, la philosophie doit nécessairement procéder par concepts $»$ (p. 109). L'Auteur conclut que le domaine de la subjectivité « relève de l'art et de la religion. Telle est, nous semble-t-il, la confusion principale de l'existentialisme » (p. 109).

Le professeur Kenneth Gallagher, de Fordham University, donne en 1963, dans la revue canadienne de philosophie Dialogue $^{18}$ un compte rendu du livre de Gabriel Marcel, Fragments philosophiques, publié, en 1962, à Louvain. Il en analyse rapidement le contenu et souligne l'importance de cette publication pour la lecture de l'œuvre marcellienne.

En 1964, la revue Dialogue publie une étude critique du livre de Kenneth Gallagher. Cette étude, signée par L.B. Geiger, de l'Université de Montréal, s'intitule : «La philosophie de Gabriel Marcel ${ }^{19}$ ». L'Auteur se dit d'avis que l'ouvrage de K. Gallagher « constitue certainement l'un des exposés les plus compréhensifs de la pensée de Marcel, l'un des plus mûrement médités » (p. 429). Il ne se contente pas, écrit-il, « de dénoncer les critiques trop simplistes dues, soit à un refus de comprendre la position de Marcel, soit à des

17. Voici la partie la plus importante de ce passage : «Le mystère, disions-nous, est capté dans le drame de la vie concrète, non par l'abstraction, mais par le recueillement. Un recueillement pas facile à décrite. Quelquefois l'auteur le définira en termes de "penser quelque chose " par opposition à "penser à quelque chose ". II paraît être fait d'un complexe d'intelligence, de volonté et de sensibilité évoluant selon un rythme progressif. C'est un acte par lequel «j'aime", "je m'engage " et me rends "disponible " à l'égard de la personne aimée ; enfin je lui demeure "fidèle », me la rendant ainsi "présente à moi-même ". Alors elle est, elle existe vraiment pour moi parce que je n'existe que pour elle. Transposez ce langage dans le domaine religieux ; transposez encore ces étapes psychologiques en fonction de l'union mystique et vous aurez quelque idée de ce qu'on entend par l'existentialisme chrétien de Gabriel Marcel. Nouvelles catégories bien proches du vocabulaire mystique d'un saint Jean de la Croix, ordonné lui aussi à exprimer la présence vécue de Dieu à l'âme par la charité " (Ibid., p. 106).

18. Kenneth Gallagher, Compte rendu de: Fragments philosophiques, dans Dialogue, vol. II, $\mathrm{n}^{\circ} 1$ (1963), p. 96-97.

19. L.-B. GeIGer, "La philosophie de Gabriel Marcel ", dans Dialogue, III, n 4 (1965), p. 426-432. 
condamnations prononcées pour raison de non-conformité à telle autre doctrine, le thomisme par exemple » (p. 429). L'Auteur prolonge les remarques critiques de K. Gallagher en analysant une phrase que Gabriel Marcel a écrite dans l'avant-propos du livre de Gallagher, à propos de l'importance de la création. Puis il conclut : "Tout l'essentiel est dit dans cet ouvrage, mesuré et ferme tout ensemble, juste et pénétrant dans l'exposé de la pensée mouvante de Marcel, précis et équitable dans la discussion des problèmes qu'elle pose » (p. 431-432).

En 1966, c'est la revue Dialogue qui publie une causerie de Maurice Merleau-Ponty, prononcée quelques années plus tôt à la maison canadienne de la cité universitaire de Paris. Ce texte a pour titre: «La philosophie de l'existence ${ }^{20} »$. Une partie de cette causerie analyse la pensée philosophique de Gabriel Marcel.

Puis, quelques articles sur Gabriel Marcel paraîtront en 1969. D'abord, un bref compte rendu à l'occasion de la réédition du tome II du Mystère de l'Être ; signé par Germain Lesage, ce compte rendu a paru dans la Revue de l'Université d'Ottawa' .

La même année, le Laval Théologique et Philosophique accepte de publier une étude du professeur américain Rudolph J. Gerber intitulée «Gabriel Marcel and the Existence of $\operatorname{God}^{22} \gg$.

La revue Dialogue donne pour sa part un article substantiel de K. Gallagher, de Fordham University : « La pensée créatrice, Marcel et Heidegger ${ }^{23} \gg$.

En 1969 également, Germaine Cromp, de l'Université de Montréal, présente aux lecteurs de la revue Dialogue une étude

20. M. Merleau-Ponty, “La philosophie de l'existence ", dans Dialogue, vol. V, $\mathrm{n}^{\circ} 3$, 1966 , p. 307-322.

21. Germain LESAGE, o.m.i., «Bibliographie ", dans Revue de l'Université d'Ottawa, vol. XXXIX, p. 322.

22. R.-J. Gerber, "Gabriel Marcel and the Existence of God ", dans Laval Théologique et Philosophique, vol. XXV, ${ }^{\circ} 1$ (1969), p. 9-22. Le professeur Gerber est docteur en philosophie de Louvain et rattaché à University of Notre Dame, Indiana.

23. K. GallaGHER, « La pensée créatrice : Marcel et Heidegger », dans Dialogue, vol. VIH, $\mathrm{n}^{\circ} 1,(1969)$ p. $22-43$. 
intitulée "Le rapport âme-corps chez le premier $\mathrm{Marcel}^{24}$ ». L'Auteur y expose le cheminement de la pensée de Gabriel Marcel sur le thème du corps-objet et du corps-sujet, établit une brève comparaison avec le même thème chez Michel Henry, souligne quelques rapprochements avec MerleauPonty, signale certaines ambiguités de la position marcellienne et annonce qu'une étude du « second Marcel» apportera une solution à ces ambiguïtés 25 .

Germaine Cromp a signé trois autres études sur la pensée de Gabriel Marcel, l'une parue en 1970 dans la Revue de l'Université d'Ottawa ${ }^{26}$, une autre en 1970 également dans Science et Esprit ${ }^{27}$ et la troisième en 1972 dans le Laval Théologique et Philosophique ${ }^{28}$. La première expose le thème de l'espérance dans un texte intitulé : "La situation immédiate de l'espérant chez Gabriel Marcel ». L'Auteur collige la pensée marcellienne autour de ce thème et fait ressortir les conditions de l'espérance authentique telles qu'on les rencontre chez le philosophe français.

La deuxième étude porte le titre suivant : «L'Avoir et l'Être dans le lien au corps chez le second Marcel ». Dans cet article, l'Auteur cerne la notion marcellienne d'incarnation et dégage à l'intérieur de cette notion, capitale chez Marcel, le rapport corps-avoir qui est ensuite approfondi.

24. Germaine CROMP, «Le rapport âme-corps chez le premier Marcel ", dans Dialogue, vol. VIII, n० 3 (1969), p. 445-459.

25. Voici les ambiguïtés soulignées par l'auteur: "Chez Gabriel Marcel, l'expression "médiation sympathique " ne rend-elle pas songeur? Le terme "sympathique " suffit-il à exprimer l'immédiateté de l'expérience ? Mais le moi global de Marcel n'est-il pas là pour escamoter la difficulté de "situer " la conscience pensante et sentante par rapport à l'esprit, de "situer " l'âme qui, d'ailleurs, semble ici conscience sentante? Tous ces «éléments " ne sont-ils pas "intégrés " dans le moi global ? Le corps-sujet alors ne serait-il pas un aspect du sujet que je suis, cet aspect couvrant l'âme ou la conscience sentante? Jusqu'à maintenant Marcel rejette cette notion du rapport âme-corps et pour le terme rapport et pour le terme âme (cf. DH 68). Pour sauvegarder l'unité du moi, Marcel ne devrait-il pas s'orienter vers la spiritualisation du corps-sujet ? Le sentir ne deviendra-t-il pas un mode de l'intelligible? L'étude du second Marcel apportera une solution à ces ambiguïtés " (lbid., p. 459).

26. Germaine Cromp, "La situation immédiate de l'espétant chez Gabriel Marcel ", dans Revue de l'Université d'Ottawa, vol. XL (1970), p. 573-584.

27. Germaine Cromp, "L'Avoir et l'Être dans le lien au corps chez le second Marcel », dans Science et Esprit, vol. XXIl, 1970, p. 49-75.

28. Germaine Cromp, «La Communion à soi-même chez Gabriel Marcel », dans Laval Théologique et Philosophique, vol. XXVIII, 1972, p. $171-184$. 
Le troisième article de Germaine Cromp articule la réflexion autour du thème " $\mathrm{La}$ communion à soi-même chez Gabriel Marcel ». Ce thème y est analysé avec ses corrélatifs d'appartenance, de créativité et de liberté. Tout au long de ces trois textes, l'Auteur, qui maîtrise manifestement bien la pensée de Gabriel Marcel, s'applique à retracer le cheminement de cette pensée cursive, à renouer les liens qu'une première lecture du philosophe ne laisse pas apercevoir, à présenter clairement aux lecteurs la philosophie marcellienne et à la leur rendre sympathique.

Il faut mentionner aussi qu'en 1972, une étude critique intitulée "Confessions philosophiques: Coleridge et Marcel », par Ernest Joos, a paru dans la revue Dialogue ${ }^{29}$. L'Auteur y analyse le livre de Gabriel Marcel, publié en 1971, sous le titre: Coleridge et Schelling. À son avis, ce livre appartient au domaine des confessions : "Quand un philosophe décide de publier son œuvre de jeunesse, dirions-nous, cela tombe dans la catégorie des confessions» (p. 602). L'Auteur manifeste sa réticence à l'endroit de la philosophie marcellienne dont il dénonce le romantisme (p. 603). « Ne retrouvons-nous pas déjà, écrit-il, dans cette œuvre de jeunesse la métaphysique particulière d'un Marcel avec son insistance sur la transcendance de Dieu qui découle de son irrationnel ? » (p. 604). Cependant, il reconnaît la valeur d'une telle problématique, à cause des possibilités qu'elle ouvre tout de même ${ }^{30}$.

Pour compléter l'inventaire des textes se rapportant à Gabriel Marcel dans les revues canadiennes, je dois mentionner les comptes rendus que j'ai donnés de trois ouvrages publiés récemment: Entretiens autour de Gabriel Marcel ${ }^{31}$, Gabriel

29. Ernest Joos, "Confessions philosophiques : Coleridge et Marcel ", dans Dialogue, vol. XI, $n^{\circ} 4$ (1972), p. 602-607.

30. Voici la critique qui termine l'arricle d'Ernest Joos : «... une métaphysique qui lance un défi à la pensée pour tourner vers une mystique y trouvera peut-être une sagesse, mais il est peu probable qu'elle y trouve plus qu'une problématique pour une métaphysique furure. Il va sans dire que "les ailes du papillon" de la métaphysique marcellienne restent "dans la peau de la chenille". Néanmoins il faut reconnaître la valeur d'une relle problématique, car elle ouvre la possibilité pour une transcendance qui fait la distinction entre ordre divin et ordre humain, entre l'inaltérable et le contingent ( $p$. 607).

31. Simonne Plourde, Compte rendu de Entretiens autour de Gabriel Marcel dans Laval Thénlogique et Philosophique, vol. XXXIII, n 3, oct. 1977, p. 314-317. 
Marcel interrogé par Pierre Boutang ${ }^{32}$, Jean Walb et Gabriel Marcel $^{33}$. Les deux premiers ont été publiés dans Le Laval Théologique et Philosopbique, le troisième dans la revue Dialogue. Le petit livre: Jean Walb et Gabriel Marcel a aussi fait l'objet d'un autre compte rendu ; ce dernier, signé par J-D. Robert, a paru dans le Laval Théologique et Philosophique ${ }^{34}$.

\section{Intérêt porté à l'auvre de Gabriel Marcel}

Les revues canadiennes ont donc publié au total, de 1941 à 1978, quatre (4) textes signés par Gabriel Marcel lui-même ; onze (11) études critiques et sept (7) comptes rendus d'ouvrages sur Gabriel Marcel ; cinq (5) études critiques sur l'existentialisme où il est question de la pensée marcellienne ${ }^{35}$.

Une trentaine de textes parus en l'espace de trente-sept ans ne constitue pas, à mon avis, une quantité suffisante pour conclure à un engouement des Canadiens à l'égard de la philosophie de Gabriel Marcel. Ces études attestent cependant que les professeurs canadiens n'ont pas ignoré le philosophe français. Ils ont jugé sa philosophie assez valable pour la présenter aux lecteurs et la critiquer. Certains (en particulier le professeur Cromp) s'y sont même franchement intéressés et se sont appliqués à l'approfondir et à la maîtriser.

\section{Accueil réservé à la philosophie marcellienne}

Au Canada français, la première lecture de la philosophie de Gabriel Marcel, tout comme celle de l'existentialisme en général, s'est effectuée en référence à la philosophie thomiste, comme le laissent percevoir avec évidence les études citées plus haut des professeurs Monette (1952), Saint-Jacques (1956) et Croteau (1959). Pour qui a connu le contexte de l'enseignement de la philosophie jusqu'aux environs de 1965, il n'y a là

32. Simonne Plourde, Compte rendu de Gabriel Marcel interrogé par Pierre Boutang, dans Laval Théologique et Philosophique, vol. XXXIV, n² 2 , juin 1978, p. 222.

33. Simonne Plourde, Compte rendu de Jean Walh et Gabriel Marcel, dans Dialogue, revue canadienne de philosophie, vol. XVII, $\mathrm{n}^{\circ} 1$, (1978), p. 180-183.

34. J.D. ROBERT, Compte rendu de Jean Walb et Gabriel Marcel, dans Laval Théologique et Philosopbique, vol. XXXIII, n 2 , juin 1977, p. 213-214.

35. Il y a sans doute d'autres études sur l'existentialisme qui ne figurent pas ici. Je n'ai pas fait le dépouillement particulier de ce thème dans les Index analytiques, faute de temps. Peut-être aurais-je repéré sous cette rubrique d'autres textes qui font référence à Gabriel Marcel? 
rien d'étonnant. Cette étude de Gabriel Marcel à la «lumière " du thomisme n'a pas porté des fruits nécessairement négatifs. On trouve une présentation positive de la pensée marcellienne et on devine une sympathie sous-jacente pour le philosophe français dans les deux études du professeur Monette $(1949,1952)$ qui ne manque pas pourtant, dans son texte de 1952, de souligner les limites qu'il décèle dans la philosophie marcellienne.

Sans référence au thomisme, les professeurs Wild (1957) et Cromp (1969, 1970, 1972) exposent la pensée de Gabriel Marcel et tentent d'en repérer le cheminement. Leur présentation est positive. Dès 1957 , le professeur Wild atteste la valeur de cette pensée et pressent la place qu'elle occupera dans l'histoire.

D'autre part, certaines études critiques émettent de sérieuses réserves à l'endroit de la philosophie marcellienne. Ainsi en font foi les textes des professeurs Pruche (1951), Croteau (1959) et Joos (1972). Leur critique dénonce, entre autres, les lacunes d'une pensée qui laisse place à l'intervention du surnaturel (Pruche), qui utilise un vocabulaire apparenté à celui de l'art et de la mystique (Croteau et Joos), qui se veut anti-rationaliste et concrète (Joos).

Voilà, dans ce mélange de sympathie et de réserve, avec ce jugement critique sérieux, l'accueil réservé par des professeurs canadiens à la philosophie de Gabriel Marcel.

\section{LES THÈSES PRÉSENTÉES DANS LES UNIVERSITÉS CANA- DIENNES}

Après avoir entendu les maîtres, nous devons nous tourner vers les étudiants. Quel intérêt ces derniers ont-ils manifesté pour l'œuvre de Gabriel Marcel et quel accueil ont-ils réservé à sa pensée?

J'ai pu repérer jusqu'à maintenant cinq (5) thèses de doctorat et trente (30) thèses de licence ou maîtrise consacrées à la philosophie et au théâtre de Gabriel Marcel. Ces trente-cinq (35) thèses ont été rédigées entre 1955 et 1975 , 
soit durant une période de vingt ans. Six (6) d'entre elles sont écrites en anglais. Faute de sources, je n'ai pu identifier les thèses qui ont été rédigées par des étudiants canadiens inscrits à des universités européennes ${ }^{36}$.

Ces thèses ont été rédigées en vue de l'obtention d'un grade en philosophie, à l'exception de deux d'entre elles, dont l'une postulait un doctorat en lettres et l'autre une maîtrise en sciences religieuses ${ }^{37}$.

Le titre de chacune de ces thèses m'a indiqué que onze (11) d'entre elles analysent soit l'aspect méthodologique, soit les notions d'être, de connaissance et de vérité dans la philosophie de Gabriel Marcel. Les vingt-quatre (24) autres thèses articulent la pensée marcellienne autour d'un thème particulier. Les thèmes abordés sont, par ordre de fréquence : l'espérance (5), la communication (4), le corps (2), la personne (2), l'amour (2), le mystère (2), la disponibilité, la fidélité, la foi, la liberté, l'engagement, la chasteté, l'éducation. Deux thèses établissent une comparaison entre Gabriel Marcel et un auteur : Marcel et Sartre, Marcel et Camus.

Deux thèses de doctorat en philosophie sont actuellement en cours à l'Université de Montréal ; l'une étudie les notions d'abstrait et de concret chez Gabriel Marcel ; l'autre compare la notion de transcendance chez Louis Lavelle et Gabriel Marcel $^{38}$.

\section{Chronologie et provenance des thèses}

La première thèse canadienne sur Gabriel Marcel indiquée dans les Index analytiques remonte à 1955. L'année 1964 fut

36. Je sais qu'il en existe au moins deux : celle de Charles Widmer, Gabriel Marcel et le théisme existentiel, présentée à l'Université de Fribourg, Suisse, et dirigée par le R.P. M.-D. Philippe; celle de Raymond SévignY, La participation chez Gabriel Marcel, présentée à l'Université de Dijon, France, et dirigée par Jeanne Parain-Vial. La première a été présentée en 1969 (ou en 1970 ?) pour l'obtention d'un doctorat en lettres ; la seconde en 1974 pour l'obtention d'un doctorat en philosophie.

37. Il s'agit des deux thèses suivantes :

Jean-Marie MassicotTe, Le théâtre de Gabriel Marcel, laboratoire du philosophe, Faculté des Lettres, Université Laval, 1968. - Marie LabrecQue, L'Espérance chez Gabriel Marcel, Faculté de Théologie, Université de Montréal, 1964. C'est par hasard que j'ai pu recenser ces deux thèses. Le temps ne m'a pas permis de dépouiller dans les Index analytiques d'autres rubriques que celle intitulée " philosophie".

38. Je tiens ces renseignements du professeur Germaine Cromp, de l'Université de Montréal. 
particulièrement prolifique avec neuf thèses consacrées à Marcel dont huit furent présentées à l'Université de Montréal ${ }^{39}$.

Sur un total de trente-cing thèses, vingt-cing (25) proviennent de l'Université de Montréal, trois (3) de l'Université Laval, trois (3) de Toronto University, trois (3) de l'Université d'Ottawa et une (1) thèse de St. Mary's University, Halifax.

\section{Contenu des thèses}

J'ai eu l'opportunité d'examiner attentivement dix-neuf (19) des trente-cinq (35) thèses colligées.

Parmi ces dix-neuf (19) thèses, une seule est consacrée exclusivement au théâtre de Gabriel Marcel. Deux (2) autres utilisent le théâtre au besoin, par la citation de répliques des personnages.

Une thèse de doctorat, celle de Hermance Baril qui développe le thème de l'engagement personnel chez Gabriel Marcel, présente l'originalité d'inclure la dimension du musicien qu'était Gabriel Marcel. L'Auteur veut montrer que la musique est l'âme de la philosophie marcellienne ${ }^{40}$. L'Auteur fait également un certain nombre de réflexions sur le théâtre marcellien, sans cependant analyser de pièces. C'est la seule thèse, parmi les dix-neuf examinées, qui englobe le Marcel philosophe, dramaturge et musicien. Les autres thèses ont choisi comme champ d'études la seule dimension philosophique chez Gabriel Marcel.

\section{Accueil réservé à la philosophie de Gabriel Marcel}

\section{A. Réserve}

Parmi les dix-neuf (19) thèses examinées, une seule oppose de sérieuses réserves à la pensée marcellienne. C'est la

39. Voir, en appendice, B) Liste des thèses.

40. Voici ce qu'écrit l'Auteur : "Originale, norre recherche l'est encore en ce qu'elle aborde la philosophie marcellienne par le biais de la musique; démarche pour laquelle nous avons reçu l'approbation de Gabriel Marcel lui-même" (p. 20). « Notre volonté est d'aller plus loin et de montrer que la musique est l'âme de sa philosophie. Nous voulons savoir pourquoi lui-même a pu dire que, dans l'expérience musicale, il a pu découvrir 'le point de départ d'une réflexion philosophique entièrement renouvelée' "(Hermance BARil, L'engagement personnel chez Gabriel Marcel, thèse de doctorat, Université de Montréal, 1968, p. 21). 
première qui apparaît en liste ${ }^{41}$. Elle fut présentée en 1955 , à l'Université de Montréal, pour l'obtention d'une maîtrise en philosophie. L'Auteur, Gerald Morgan, critique les fondements épistémologiques de la métaphysique marcellienne à la lumière des catégories de la philosophie thomiste, avec références à Maritain. Il compare la pensée thomiste avec la pensée marcellienne et établit des affinités et des divergences entre Marcel et Maritain. À son avis, Gabriel Marcel se livre davantage à la mystique et à l'art qu'à la métaphysique. Les difficultés fondamentales que présente pour lui la philosophie marcellienne proviennent, d'une part, du recours à la transcendance (qui appartient au domaine de la mystique) ${ }^{42}$ et, d'autre part, à l'usage de la métaphore, à la place de l'abstraction, pour communiquer l'expérience (ce qui appartient au domaine de l'art) ${ }^{43}$. G. Morgan constate que $G$. Marcel a opéré un choix judicieux en confiant à la musique et au drame le soin d'exprimer cette sorte d'expérience très importante pour une personne de son tempérament. Mais, dit-il en substance, cela n'a rien à voir avec la métaphysique telle que comprise par des étudiants de la philosophia perennis ${ }^{44}$. Au dernier chapitre de sa thèse, l'Auteur montre que la philosophie de Gabriel Marcel fait usage des termes utilisés par l'art dramatique : les termes "présence», «mystère», "participation».

Cette lecture de Gabriel Marcel rejoint celle que nous avons rencontrée chez les mâtres à la même période.

\section{B. Critique}

Trois (3) thèses exposent la philosophie marcellienne et tout en l'acceptant dans l'ensemble, elles formulent des objections.

Marie Gérin-Lajoie ${ }^{45}$, après s'être dit d'accord avec le rejet de l'abstraction effectué par Marcel, s'oppose à sa

41. Gerald Morgan, Connotational tinking in the philosopby of Gabriel Marcel, thèse de maîtrise, Université de Montréal, 1955, 58 p.

42. Ibid., p. 17.

43. Ibid., p. 20-22.

44. Ibid., p. 27.

45. Marie Gerin-Lajoie, Recherche sur la connaissance dans la pbilosopbie de Gabriel Marcel, thèse de licence en philosophie, Université de Montréal, 1959, 105 p. 
condamnation aveugle de l'objectivité. L'Auteur recourt à quelques distinctions. thomistes pour appuyer ses objections. En conclusion, elle pose ce jugement sympathique : "Finalement, il nous semble que Marcel a eu, le premier depuis longtemps dans le monde philosophique, la vision profonde de vouloir réapprendre aux hommes l'admiration et la contemplation qui sont à la source de toute sagesse ${ }^{46}$."

Dans sa thèse de doctorat, Thérèse Gingras ${ }^{47}$ expose la pensée marcellienne sans en établir une critique poussée. Elle pose en terminant une question à Gabriel Marcel : "Comment expliquer qu'une même méthode d'approche dite "phénoménologie existentielle » conduise à l'" invocation adoratrice du Toi absolu ou à la suprême «néantisation » de l'Être absolu ? $^{48} »$. Elle conclut, en se ralliant à l'optique marcellienne : «La foi demeure un mystère ontologique 'à l'articulation de la liberté et de la grâce' » (p. 316).

La thèse que j'ai moi-même consacrée à La métaphysique de l'espérance chez Gabriel Marcel ${ }^{49}$ tente d'unifier la pensée du philosophe autour du thème de l'espérance. La pensée marcellienne a toujours suscité chez moi malgré ses limites un vif intérêt et trouvé un accueil positif. J'ai signalé dans mon étude les limites que j'ai perçues dans cette philosophie concrète qui ne porte guère attention à l'être infra-humain, qui se heurte à une conception idéaliste des notions d'objet et de cause, qui n'utilise pas l'analogie (instrument important en ontologie) et dont la certitude propre est plutôt de l'ordre de l'affirmation et du témoignage que de celui de la démonstration.

\footnotetext{
46. Ibid., p. 102 .

47. Thérèse Gingras, Le mystère de la foi dans la philosophie existentielle de Gabriel Marcel, thèse de doctorat en philosophie, Montréal, 1966, 352 p.

48. Ibid., p. 314. Voici deux autres passages qui explicitent sa question : "Les existentialistes se situent au plan métaphysique. N'opèrent-ils pas le passage d'une phénoménologie de l'existence à une métaphysique de la contingence ? " (p. 315). «Ce ne serait donc pas la méthode phénoménologique comme telle qui nierait ou affirmerait la réalité de l'être et la présence de l'absolu. Ne serait-ce pas plutôt l'ontologie " néantisante » ou "créatrice", juxtaposée à la phénoménologie? » (p. 315 ).

49. Simonne Plourde, La métaphysique de l'espérance chez Gabriel Marcel, thèse de doctorat en philosophie, Université Laval, 1972, $515 \mathrm{p}$.
} 


\section{Exposé}

Les quinze (15) autres thèses dont $j$ 'ai pris connaissance offrent chacune un exposé de la philosophie de Gabriel Marcel. Elles s'efforcent de comprendre cette pensée sinueuse et de l'articuler le plus clairement possible autour du thème choisi. Elles révèlent chez les auteurs un accueil sympathique réservé à la pensée marcellienne. En voici deux exemples:

Notre contemporain n'est plus l'homme de Descartes, mais bien plutôt l'homme de Pascal, homme jeté dans l'univers, avec sa grandeur et sa misère, problème pour lui-même et pour les autres. Notre contemporain n'a jamais autant aspiré à la communion. Il voudrait rompre la solitude. Cette exigence nous est apparue comme caractérisant la pensée de Gabriel Marcel $^{50}$.

La philosophie de Gabriel Marcel est incomplète, et cela tient pour une part à l'ampleur et aux difficultés inhérentes à son entreprise. Mais le but qu'il s'est proposé avant tout, c'est "d'ouvrir l'esprit et de l'engager dans une recherche personnelle . Sa pensée demeure vivante, ouverte, et ne demande qu'à être prolongée. Comme il le dit lui-même, elle introduit sur une « voie difficile mais libératrice ${ }^{51}$ ».

Hermance Baril, dans la thèse citée plus haut, a aussi exprimé sa sympathie envers la pensée marcellienne:

L'être engagé marcellien rejoint donc l'homme contemporain, centre de participations multiples et point de rencontre de communautés diverses, qui ne s'épanouit que dans l'acceptation de responsabilités nombreuses (p. 21).

Nous croyons que tout en captant les mouvements de l'époque, l'engagement marcellien sauvegarde deux valeurs essentielles à l'humanisme contemporain : la créativité et la fraternité ( $p$. 312).

Je pourrais poursuivre les citations. Celles-ci me paraissent suffisantes pour appuyer ma conclusion : la pensée de Gabriel Marcel a suscité l'intérêt des étudiants canadiens et elle

50. Charles Gerva1s, Vers une philosophie de la communication, thèse de licence en philosophie, Université de Montréal, 1962, 104 p. (p. 100).

51. Richard Bordeleau, La démarche métaphysique de Gabriel Marcel et son enracinement existentiel, thèse de licence en philosophie, Université de Montréal, 1967, 236 p. (p. 233). 
a rencontré chez eux, tout au moins dans l'ensemble des thèses que j'ai pu lire, un accueil favorable. Les deux critères posés au départ du nombre des thèses et de la valeur reconnue à la philosophie de Gabriel Marcel par chacune autorisent cette conclusion.

\section{LES LIVRES CONSACRÉS À L'OEUVRE DE GABRIEL MARCEL}

Si les thèses présentées dans les universités canadiennes sont relativement nombreuses, rares sont celles qui ont été publiées. Quelques étudiants canadiens ont poursuivi leurs études dans les universités européennes et leur thèse a été éditée à l'étranger. Il ne m'a pas été possible d'en faire un relevé exhaustif. Voici les maigres résultats de ma cueillette.

A. Sur la philosophie de Gabriel Marcel: Gérard Bélanger, L'Amour, chemin de la liberté ; essai sur la personnalisation (Paris, les Éditions ouvrières, 1965, 181 p.). - Charles Widmer, Gabriel Marcel et le théisme existentiel (Paris, Éd. du Cerf, 1971, 244 p.). - Simonne Plourde, Gabriel Marcel, philosophe et témoin de l'espérance, (Montréal, P.U.Q., 1975, 232 p.).

B. Sur l'existentialisme: F.T. Kingston, French Existentialism. A Christian critique, (Toronto, University of Toronto Press, 1961, 221 p.).

C. Articles dans un collectif : Jacques Croteau, Une philosophie de la communion: Gabriel Marcel ${ }^{52}$. - Jean Theau consacre quelques pages à Gabriel Marcel dans son livre : La philosopbie française dans la première moitié $d u X X^{e}$ siècle ${ }^{53}$.

4. ÉMISSIONS RADIOPHONIQUES, JOURNAUX, CERCLES ET ASSOCIATIONS

Pour compléter cette revue sur la présence de Gabriel Marcel au Canada, je dois signaler la visite de Gabriel Marcel

52. Yvon Lafrance et John King-Farlow, eds., La philosophie et les philosophes, tome II, Montréal, Les Éditions Bellarmin ; Paris-Tournai : Desclée, 1973, p. 207-231.

53. La Pbilnsophie française dans la première moitié du XX siècle, Ottawa, Éd. de l'Université d'Ottawa, Coll. "Philosophica ", 1977. 
en 1956, quelques articles parus dans les journaux relativement au philosophe, les cercles et les associations qui diffusent la pensée marcellienne.

\section{A. Gabriel Marcel au Canada}

Invité par la Société d'Études et de Conférences de Montréal ${ }^{54}$, Gabriel Marcel venait au Canada en 1956. Dans son numéro du 7 avril, la Semaine à Radio-Canada consacrait un article à Gabriel Marcel et annonçait une interview du philosophe à Conférence de presse, émission simultanément radiodiffusée et télédiffusée le samedi, 9 avril ${ }^{55}$. L'article mentionnait en outre la série de causeries qui seraient données par Gabriel Marcel au réseau français de Radio-Canada.

Gabriel Marcel a écrit, par la suite, deux autres textes pour la radio canadienne $: \grave{A}$ propos de la bombe atomique (janvier 1960); La philosopbie de l'existence (février 1961) ${ }^{56}$.

\section{B. Les journaux}

Dans son numéro du 21 mars 1969, le Devoir reproduisait un article du journal français Le Monde, dû à la plume du philosophe Étienne Borne et intitulé : « Maîtres de la sagesse : la pensée de Gabriel Marcel ».

Le 28 août 1971, La Presse publiait un article de Cécile Brosseau intitulé : "Adeptes de la pensée de Gabriel Marcel au congrès des sociétés de philosophie ».

Le Devoir du 9 octobre 1973, annonçait aux lecteurs canadiens la mort de Gabriel Marcel survenue la veille à Paris.

54. J'ai retrouvé, aux Archives Gabriel Marcel, à Paris, les titres des dix conférences que G. Marcel a prononcées au Canada. Les voici : - La croyance comme dimension spirituelle - Religion et blasphème dans le théâtre français contemporain - Justification et limites de la philosophie existentielle - La fonction spirituelle du thêâtre - Le crépuscule du sens commun - L'être devant la pensée interrogative - Mon itinéraire philosophique - Les différentes tendances du théâtre d'aujourd'hui - Pourquoi j'ai écrit pour le thêâtre - Hommage à Charles Du Bos.

55. C'est à la suite de cerre émission que A. ST-Jacques a publié " $\grave{A}$ propos d'une affirmation de M. Gabriel Marcel ", dans la Revue de l'Université Laval. Voir note 14.

56. Cf. Roger Trolsfontaines, De l'Existence à l'Être. Paris-Louvain, Nauwelaerts, tome II, p. 463 et 464 . 


\section{Les Cercles et les Associations}

Le professeur Alexis Klimov, de l'Université du Québec à Trois-Rivières, a fondé, il y a quelques années, le Cercle Gabriel Marcel qui vise à faire connaître la pensée du philosophe. Très vivant, le Cercle trifluvien regroupe mensuellement de soixante à quatre-vingts personnes. À chaque rencontre, on offre un exposé sur un thème marcellien, suivi d'un échange avec les participants ${ }^{57}$.

L'Association "Présence de Gabriel Marcel », créée à Paris en 1975, compte actuellement plus de quatre cents (400) membres, de vingt et un pays différents. Font partie de cette Association une vingtaine de membres canadiens; ce nombre situe le Canada au second rang, immédiatement après la France. L'Association internationale vise à prolonger la présence de Gabriel Marcel et à diffuser sa pensée ; elle publie annuellement un Cahier Gabriel Marcel.

Au terme de cette recherche qui visait à identifier la présence de Gabriel Marcel au Canada, je crois pouvoir affirmer que les Canadiens, et en particulier les Québécois, manifestent de l'intérêt pour l'œuvre de Gabriel Marcel. La philosophie marcellienne fait l'objet d'études de la part de professeurs et d'étudiants, ainsi que nous avons pu le constater. Le grand public commence à être rejoint par le truchement des publications et des cercles. L'accueil réservé à cette pensée est pondéré par une critique sérieuse chez les professeurs. Les étudiants et le public sont rejoints par les valeurs contemporaines de créativité, de fraternité, de communion, d'espérance que leur présente la philosophie de Gabriel Marcel.

Au «Colloque Gabriel Marcel » de Cerisy-la-Salle, en août 1973, le professeur allemand Vincent Berning affirmait : «Malgré les limites qui ont marqué jusqu’à présent les virtualités d'un accueil fait en Allemagne à la pensée marcellienne, je sais par mon expérience de professeur en contact avec des étudiants que cette pensée est jeune et qu'elle a un avenir,

57. Je tiens ces informations de M. Klimov. 
en Allemagne aussi ${ }^{58}$. $\gg$ Mon propre contact avec les étudiants me permet de formuler la même affirmation et de croire à un avenir pour la philosophie marcellienne au Canada.

Université du Québec à Rimouski

58. Vincent BERNING, "Données et Conditions de l'accueil fait en Allemagne", dans Entretiens autour de Gabriel Marcel, La Baconnière, Neuchatel, 1976, p. 219. 


\section{APPENDICE}

\section{A. Sources utilisées}

Je tiens à remercier vivement madame Yolande Thwaites qui, pour les fins de cette recherche, a effectué le dépouillement des sources suivantes :

- Bibliographie du Québec, 1968-1977 incl.

- Bibliographie d'Histoire littéraire française, 1956-1976.

- Cahiers bibliographiques des Lettres québécoises, 19661969.

- Canadiana, 1950-1978 (fév. incl.).

- Canadian Books in Print, 1967-1977.

- Canadian Book Review Annual, 1975 et 1976.

- Canadian Journal of Philosophy, Guelph, Ontario, 19711976.

- Canadian Journal of Philosophy, Guelph, Ontario, 1975 et 1976.

- Canadian Periodical Index, 1948-1977.

- Catalogue de l'Édition au Canada français, 1965-1971.

- Cirpho, 1973-1976.

- Dialogue, 1962-1977.

- French VII, 1940-1948.

- Index analytique, 1966-1972.

- Index de l'Actualité, 1972-1977.

- Laval Théologique et Philosophique, 1945-1978 (juin incl.).

- Livres et Auteurs Québécois, 1961-1976.

- Mill News Letter, Toronto, 1965-1976.

- MLA International Bibliography, 1921-1925, 1972-1975.

- Periodex, 1972-1978 (fév. incl.).

- Philosophiques, 1974-1977.

- Phi zero, 1973-1977.

- Radar, 1972-1977.

- Répertoires des thèses, 1970-1975. 
- Répertoires des thèses :

- Répertoire des thèses littéraires, 1969-1971.

- Guide bibliographique des thèses littéraires canadiennes, de 1921 à 1969.

- Inventaire des recherches en cours dans les humanités, 1972.

- Thèses des gradués canadiens dans les humanités et les sciences sociales.

- Thèses canadiennes, 1947-1969.

- Revue de l'Université d'Ottawa, 1931-1976.

- Russell : The Journal of the Bertrand Russell Archives, Hamilton, Ontario, 1971-1975.

- Société de Philosophie du Québec, Bulletin, 1974-1978 (avril incl.).

B. Liste des thèses répertoriées

L'astérisque indique les thèses que j'ai pu me procurer pour en faire la lecture.

- AUDY, André, De la participation du moi au corps et à son prolongement, thèse de licence en philosophie, Université de Montréal, 1964, XX-68 p.

—*BARIL, Hermance, L'engagement personnel chez Gabriel Marcel, thèse de D. PH., Université de Montréal, 1968, 376 p.

- BLAIR, André, L'opposition de la personne au "on " selon Gabriel Marcel d'après Du Refus à l'Invocation, thèse de licence en philosophie, Université de Montréal, 1967, $125 \mathrm{p}$.

- BonNelly, Claude, Camus et Marcel : examen comparé de leur méthode face à l'inquiétude et à l'absurde, thèse de licence en philosophie, Québec, Université Laval, 1968.

-*BORDELEAU, Richard, La démarche métaphysique de Gabriel Marcel et son enracinement existentiel, thèse de licence en philosophie, Université de Montréal, 1967, 236 p.

- Boisclair, Normand, Vers l'interprétation d'un réalisme existentiel de la philosopbie de Gabriel Marcel, thèse de licence en philosophie, Université de Montréal, 1966. 
—*Bulger, D. Michael, Problem and mystery ; a fundamental distinction in the philosophical writings of Gabriel Marcel, thèse de M.A., Halifax, St. Mary's, 1967.

- ${ }^{*}$ Charbonneau, François, Recherches sur la notion de disponibilité dans la philosophie de Gabriel Marcel, thèse de licence en philosophie, Université de Montréal, 1964, $148 \mathrm{p}$.

- Charbonneau, Guy, Essai sur l'espérance, thèse de licence en philosophie, Université de Montréal, 1962.

- Charron, Ghislain, L'ontologie phénoménologique de Gabriel Marcel, thèse de M.A., Université d'Ottawa, 1964.

- CHENe, Adèle, Nos rapports avec autrui. L'autre présent et connu. Vers le conflit ou la rencontre, thèse de licence en philosophie, Université de Montréal, 1962.

- Daneau, Paul, Le corps chez Gabriel Marcel, thèse de licence en philosophie, Université de Montréal, 1964.

- Delorme, Robert. Vers la communication espérance. Défi. Inquiétude. Patience et désespoir. Amour. Espérance, thèse de licence en philosophie, Université de Montréal, 1964, 72 p.

—*Des Groseillers, Pierre, La fidélité dans l'auvre " $\hat{E}$ tre et Avoir " de Gabriel Marcel, thèse de licence en philosophie, Université de Montréal, 1964, 87 p.

—*Des Roches, Philippe, Les conditions ontologiques de la communication chez Gabriel Marcel, thèse de licence en philosophie, Université de Montréal, 1964, 86 p.

—*Desrochers, Claude, Critique de la prétention sous-jacente à la pensée pure et abstraite dans les cuvres philosophiques de Gabriel Marcel, thèse de licence en philosophie, Université de Montréal, 1966, 255 p.

- Duclos, Gilles, Savoir et technique chez Gabriel Marcel, thèse de M.A., Université d'Ottawa, 1972, 117 p.

- Evans, Elisabeth Mary, The problem of love and communication in Sartre and Marcel, thèse de M.A., Toronto University, 1965. 
-*GÉRIN-LAJOIE, Marie, Recherche sur la connaissance dans la philosopbie de Gabriel Marcel, thèse de licence en philosophie, Université de Montréal, 1959, 105 p.

- ${ }^{*}$ GervaIs, Charles, Vers une philosopbie de la communication ; essai sur la philosopbie de Gabriel Marcel, thèse de licence en philosophie, Université de Montréal, 1962, 104 p.

- GINGRAS, Thérèse, Le mystère de la foi dans la philosophie existentielle de Gabriel Marcel, thèse de D. PH., Université de Montréal, 1966, 352 p.

- LABReCQUe, Marie, (Sr Isabelle-du-Rosaire), L'espérance chez Gabriel Marcel, thèse de maîtrise en sc. religieuses, Université de Montréal, 1964, XIV-84 p.

- LAUzON, Roger, La doctrine de la personne chez Gabriel Marcel et ses implications dans une philosopbie de l'éducation, thèse de maîtrise en philosophie, Université de Montréal, $1959,47 \mathrm{p}$.

—*LePAGe, Daniel, L'amour chez Gabriel Marcel, thèse de licence en philosophie, Université de Montréal, 1966, 87 p.

- MAC NeIL, Claude, The philosopby of Gabriel Marcel and contemporary emphasis in education, Université d'Ottawa, 1969.

- Martineau, Alain, La Genèse du "Qui suis-je » chez Gabriel Marcel, thèse de maîtrise en philosophie, Université de Montréal, 1975.

- MassicotTe, Jean-Marie, Le théâtre de Gabriel Marcel, laboratoire $d u$ philosophe, thèse de D.E.S. en Lettres, Québec, Université Laval, 1968, 136 p.

- MÉnard, Yolande, Approches de la notion de vérité chez Gabriel Marcel, thèse de licence en philosophie, Université de Montréal, 1962, 114 p.

-*Morgan, Gerald-A., Connotational thinking in the philosophy of Gabriel Marcel, thèse de M.A., Université de Montréal, 1955, 58 p. 
- NOwOTNY, Joan, Freedom and being in the thought of Gabriel Marcel, thèse de M.A., Toronto University, 1966.

- NowotNy, Joan, Gabriel Marcel's philosophy of bope, thèse de PH. D., Toronto University, 1974, $371 \mathrm{p}$.

- Plourde, Simonne, La métaphysique de l'espérance chez Gabriel Marcel, thèse de D. PH., Québec, Université Laval, 1972, 515 p.

—*Rodrigue, Réal, Le rapport de la pensée et du sentir dans la philosophie concrète de Gabriel Marcel, thèse de licence en philosophie, Université de Montréal, 1964, 58 p.

- *Serres, Jeannine, Pbilosophie de la chasteté chez Gabriel Marcel, thèse de D. PH., Université de Montréal, 1970, $353 \mathrm{p}$.

- Vallières, Guy, Genèse de la notion de mystère dans Être et Avoir de Gabriel Marcel, thèse de M.A., Université de Montréal, 1969, $155 \mathrm{p}$.

N.B. : S'il y a des oublis dans cette liste, je m'en excuse et je serai reconnaissante envers qui me les signalera. 